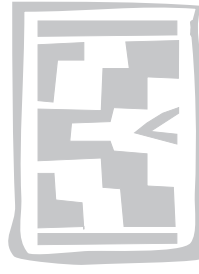

\title{
The Brody effect induced by premature ventricular complexes in the ovine heart
}

\author{
J. KER ${ }^{1 *}$, E.C. WEBB ${ }^{2}$ and D. VAN PAPENDORP ${ }^{1}$
}

\begin{abstract}
KER, J. WEBB, E.C. \& VAN PAPENDORP, D. 2009. The Brody effect induced by premature ventricular complexes in the ovine heart. Onderstepoort Journal of Veterinary Research, 76:443-448

The QRS response of the electrocardiogram to bleeding has been a source of interest to the physiologist for more than a century. Studies in the dog, cat and chicken have shown a reduction in QRS amplitude in response to bleeding. This effect has been explained by the so-called Brody effect, in which the intraventricular mass of blood acts as a conducting medium, augmenting radial conduction, thus resulting in the subsequent reduction in QRS amplitude in conditions where the intraventricular mass of blood is reduced.

The aim of this study was to evaluate whether the Brody effect will be present in the ovine heart and, furthermore, to evaluate if the right and left ventricles will demonstrate the same QRS change if the Brody effect is indeed present. This study clearly demonstrated that the Brody effect is present in the ovine heart. Furthermore, two unique aspects emanating from this study are firstly the fact that this is the first study to show that premature ventricular complexes are able to induce the Brody effect and, secondly that there is a very clear difference in the response of the right and the left ventricles when the Brody effect is induced in the ovine heart.
\end{abstract}

Keywords: Brody effect; ovine heart; QRS amplitude; premature ventricular complex

\section{INTRODUCTION}

The variations in the QRS amplitude of the electrocardiogram, caused by alterations in ventricular filling, have been a source of interest to the physiologist since 1910 (Brody 1956; Nelson, Lange, Hecht, Carlisle \& Ruby 1956; Ishikawa, Berson \& Pipberger 1971; Manoach, Gitter, Grossman \& Varon 1971; Manoach 2000). The experiments in which ventricu-

* Author to whom correspondence is to be directed. E-mail: jker@wol.co.za

1 Department of Physiology, Faculty of Medicine, University of Pretoria, South Africa

2 Department of Animal and Wildlife Sciences, Faculty of Natural and Agricultural Sciences, University of Pretoria, South Africa

Accepted for publication 16 April 2009-Editor lar filling has been increased and decreased were described in both poikilotherms and homeotherms (Manoach, Gitter, Grossman \& Varon 1972). In all these experiments, ventricular filling was decreased directly by bleeding or clamping of the inferior vena cava, or indirectly by compression of the heart via the induction of a pericardial effusion, or increased directly via the infusion of blood and other fluids or indirectly via clamping of the aorta.

In both poikilotherms and homeotherms the QRS amplitude of the electrocardiogram changes during bleeding-leading to emptying of the ventricle-or filling of the ventricle (Manoach et al. 1972).

Studies in homeotherms (normal mammals, specifically cats and dogs, as well as non-mammals, such as chickens) have shown that a decrease in ventricular filling causes a reduction in the amplitude of 
the QRS complex while increased ventricular filling causes an increase in the amplitude of the QRS complex (Manoach, Gitter, Grossman \& Varon 1972; Manoach, Varon, Grossman, Gitter \& Sroka 1971; Manoach, Gitter, Grossman, Varon \& Gassne 1971). Schwan 1928 (cited by Brody 1956) stated that the conductivities of the lung and myocardium are almost the same, but that the conductivity of intraventricular blood is approximately ten times that of the surrounding tissue and thus that the intraventricular blood mass will exert an effect upon electrical potentials generated within the myocardium. Brody (1956) examined this effect and concluded that the net effect of the intracavitary blood mass on the cardiac dipole is to augment the radial components and to reduce the tangential components, thus explaining why bleeding will lead to a reduction in the QRS amplitude in the mammalian heart. Since the original study by Brody (1956) this phenomenon became known as the Brody effect. This effect results from the lower resistivity of the intraventricular blood mass as compared to the surrounding tissues, in essence causing a short-circuiting effect (Ishikawa 1976).

However, the QRS amplitude increases after bleeding in frogs, turtles and lizards (poikilotherms) (Manoach et al. 1971; Pipberger, Ishikawa \& Berson 1972). This opposite QRS reaction to bleeding in homeotherms and poikilotherms is ascribed to phylogenetic differences (Manoach et al. 1972). However, especially in light of the Brody effect, this opposite reaction has never been properly explained in the literature to date.

There is ample experimental evidence for the Brody effect from a number of other researchers (Angelakos \& Gokhan 1963; Horan, Andreae \& Yoffee 1961; Nelson et al. 1956).

More interestingly, and poorly examined until now, are the different QRS responses of the right and left ventricles to bleeding. Angelakos \& Gokhan (1963), in their experiments on dogs, noted a decrease in amplitude of left ventricular QRS complexes to bleeding, as expected. However, they also noted an opposite effect over the right ventricle-this makes physiological sense as right ventricular activation is predominantly tangential (Pipberger et al. 1972).

Other means of altering left ventricular volume and inducing the Brody effect, such as rapid atrial pacing, have also been described (Daniels, Iskandrian, Hakki, Kane, Bemis, Horowitz, Greenspan \& Segal 1984).

The purpose of this study was to identify the existence of the Brody effect in the ovine heart. No evi- dence of any description of the Brody effect in the ovine heart could be found in the literature. Furthermore, we specifically sought to examine the possibility that the right and left ventricle will show a different QRS response during the Brody effect.

Premature ventricular complexes (PVCs) were chosen to reduce left and right ventricular volumes, in order to try and induce the Brody effect and to document any possible difference between right and left ventricular QRS amplitude responses. A premature ventricular complex (PVC) is the expression of an impulse that arises prematurely in an ectopic ventricular focus and can originate in the specialized conduction tissue distal to the bifurcation of the bundle of His or in the ventricular myocardium itself (Schamroth 1980; Myerburg \& Kessler 1998).

As a PVC by definition arises prematurely, the premature contraction of the ventricle will impair the diastolic filling and thus volume, as the diastolic time interval is shortened and will thus lead to a lower ventricular volume. The premature impulse, whether it originates from the left or right ventricle, will be propagated to the other ventricle, therefore both ventricles will contract prematurely, resulting in a lowering of both ventricular volumes. It was postulated that the Brody effect in the ovine heart could be demonstrated in this way, and it was hoped that these results would shed light on a possible different QRS response from the right and left ventricles.

\section{MATERIALS AND METHODS}

This study was performed with the approval of, and adherence to, the guidelines of the Pretoria Biomedical Research Centre's Animal Use and Care Committee.

A clinically normal Dorper wether, aged 10 months and weighing $35 \mathrm{~kg}$ was used for this study. The sheep was fed lucerne hay ad libitum and received $300 \mathrm{~g}$ per day of pelleted concentrate (10 MJ ME/kg DM with $14 \%$ crude protein) and had free access to water at all times.

After an overnight fast, the wether was sedated by an intramuscular dose of ketamine hydrochloride (Brevinaze, manufactured by Intramed) at a dose of $100 \mathrm{mg}$ and placed in the left lateral decubitus position. It was then placed under continuous electrocardiographic monitoring as follows: Einthoven's triangle was moved from the frontal to the sagittal plane, as described before by Schultz \& Pretorius (Schulz \& Pretorius 1972), by moving the standard and unipolar limb electrodes as follows: 
(a) aVR moved from the right forelimb to the head between the ears;

(b) aVL moved from the left forelimb to the sacrum;

(c) aVF moved from the left hindlimb to the sternal angle; and

(d) the earth electrode was placed on the right hindleg, just above the hock.

The six precordial leads were placed as follows, as described before by Ker \& Webb (2003):

(a) V1 placed $7 \mathrm{~cm}$ to the right of the sternal angle;

(b) V2 placed $7 \mathrm{~cm}$ to the left of the sternal angle;

(c) V3 placed $4.5 \mathrm{~cm}$ below and $1 \mathrm{~cm}$ to the left of $\mathrm{V} 2$;

(d) V4 placed $4.5 \mathrm{~cm}$ below and $1 \mathrm{~cm}$ to the left of V3;

(e) V5 placed $4.5 \mathrm{~cm}$ below and $1 \mathrm{~cm}$ to the left of $\mathrm{V} 4$; and

(f) V6 placed $4.5 \mathrm{~cm}$ below and $1 \mathrm{~cm}$ to the left of V5.

Meditrace 200 (MTD), disposable ECG conductive, adhesive electrodes were used. The skin areas where the ECG electrodes were placed were clipped and the attached electrodes were secured with Super Glue (by Bostik).

\section{Premature ventricular complexes were then induced from the left ventricular apex}

Using the Seldinger technique a spring-wire guide, diameter $0.81 \mathrm{~mm}$ and length $60 \mathrm{~cm}$, was advanced into the left ventricular apex via the right internal carotid artery under fluoroscopic guidance. For a period of $20 \mathrm{~min}$ the spring-wire guide was moved manually in order to induce PVCs from the left ventricular apex. Twelve-lead electrocardiograms were continuously recorded during this period.

\section{RESULTS}

Fig. 1 shows the 12-lead electrocardiogram of the sedated wether in the left, lateral decubitus position, before induction of PVCs. Note the negative polarity of the QRS complex in Lead V1, with an amplitude of $0.4 \mathrm{mV}$, and also the negative polarity of the QRS complex in Lead V6, with an amplitude of $1.0 \mathrm{mV}$.

Fig. 2 shows the 12-lead electrocardiogram at the start of PVC induction. Note the 3 PVCs in Lead V1. They are easily recognized by their positive QRS

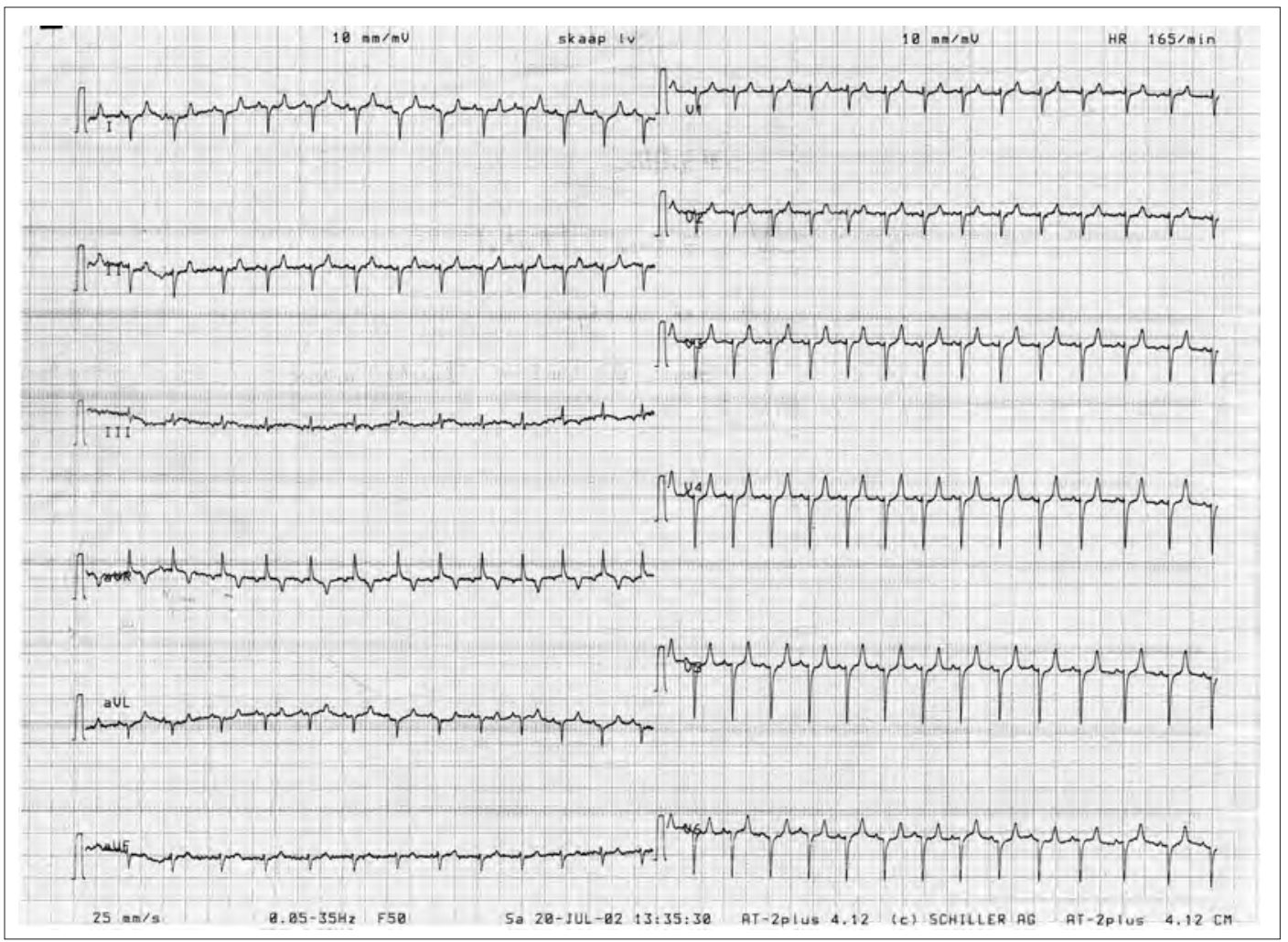

FIG. 1 The normal 12-lead electrocardiogram, before the induction of PVCs 


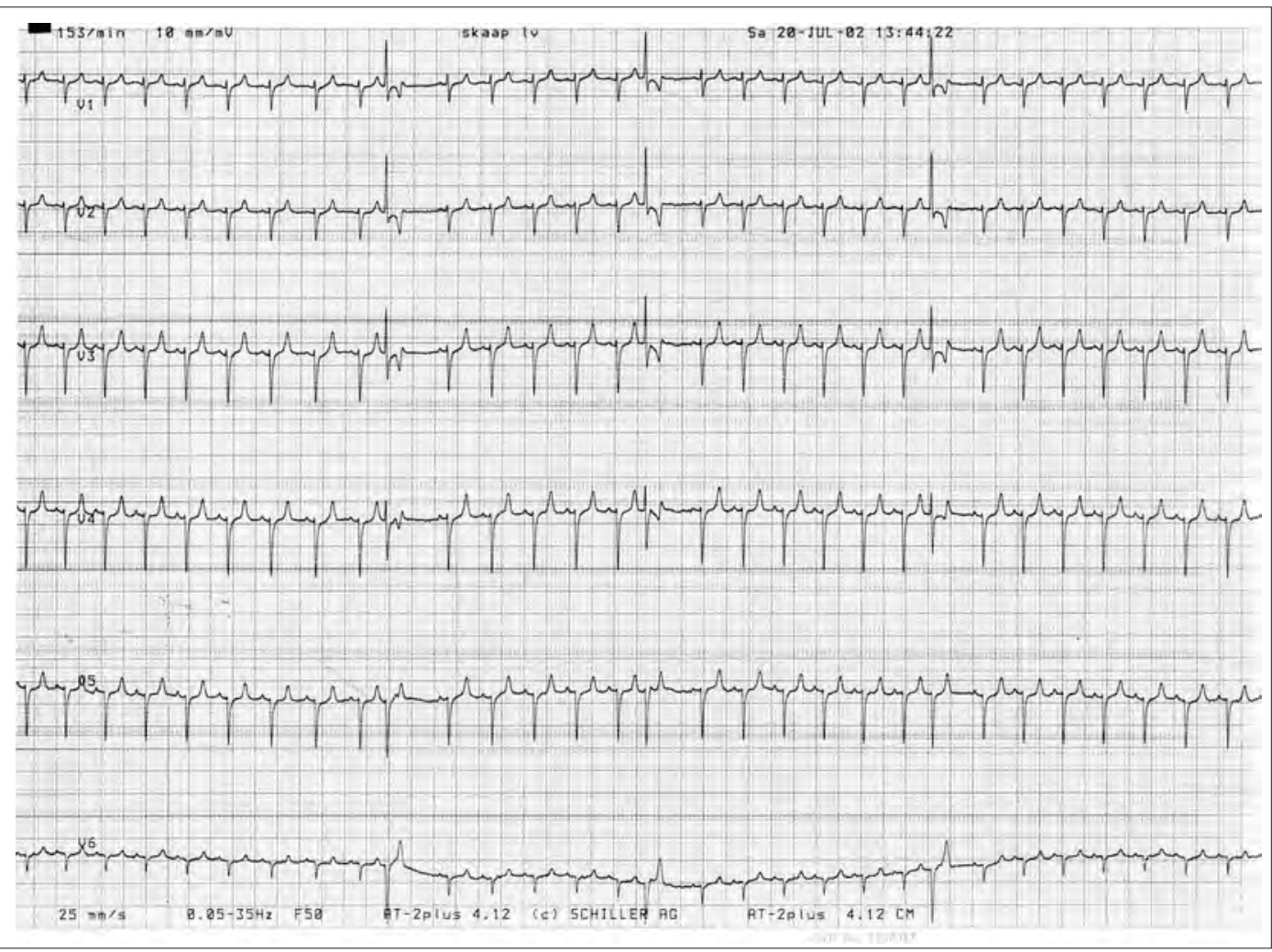

FIG. 2 Note the 3 PVCs with a positive QRS polarity in Lead V1 and a negative polarity in Lead V6

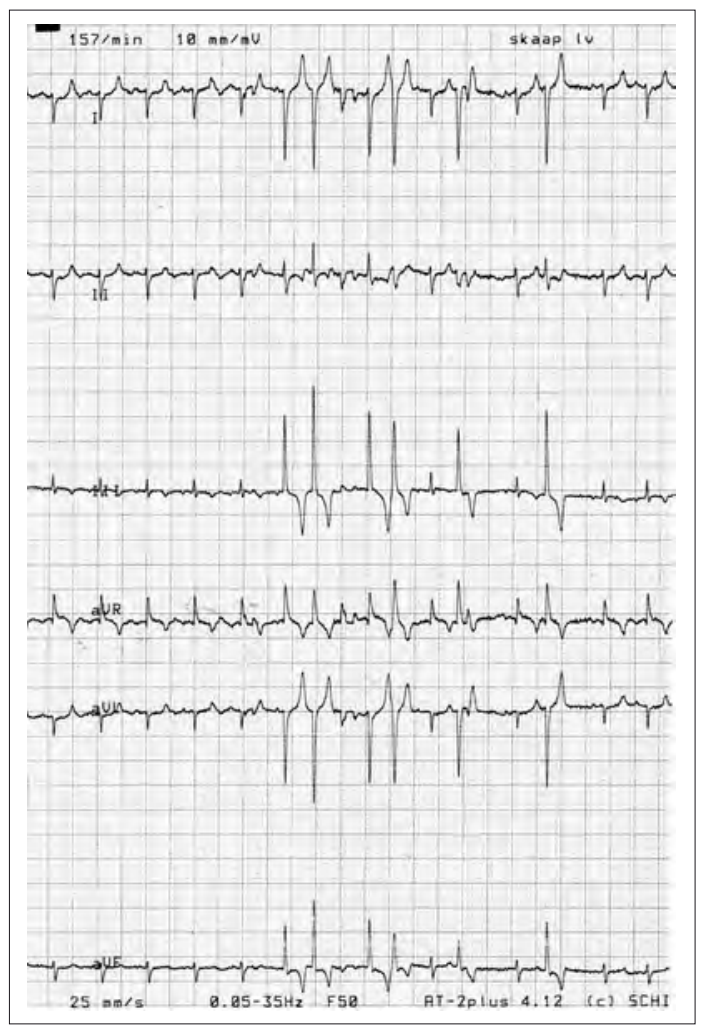

FIG. 3 The 12-lead electrocardiogram, demonstrating the appearance of PVCs in Leads I, II, III, aVR, aVL and aVF 


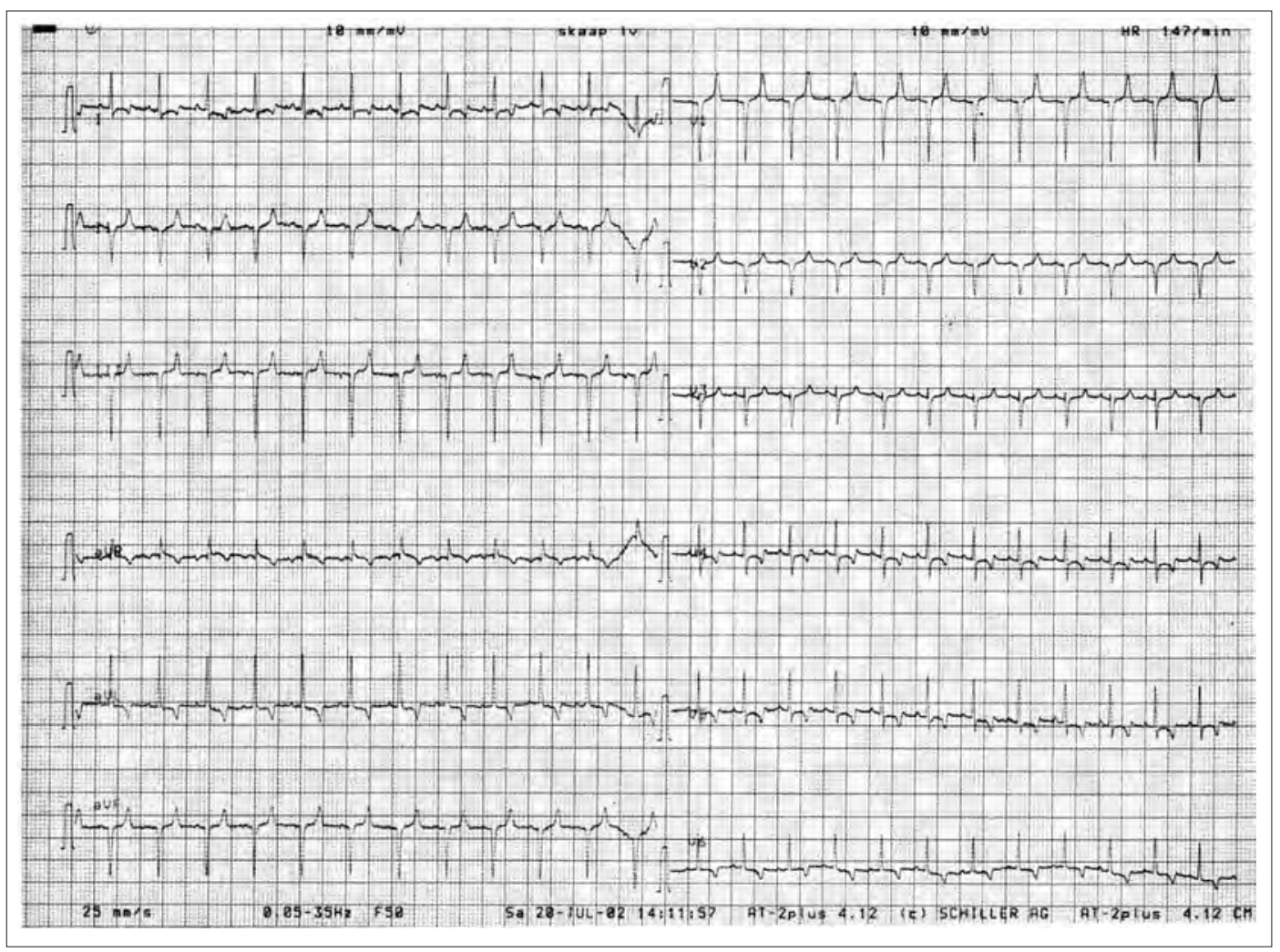

FIG. 4 This is the exact same 12-lead electrocardiogram after 28 min of PVCs. Note the increased amplitude in Lead V1 and the change in polarity of the QRS complex in Lead V6

polarity, as opposed to the rest of the normal beats. In addition, note the same 3 PVCs in Lead V6. Here they have a negative QRS polarity with a much greater amplitude than that of the normal beats. This is a very important observation, as it indicates that the chosen position for Lead V1 is ideal for evaluating the right ventricle and that the chosen position for Lead V6 is ideal for evaluating the left ventricle: The PVC originates from the apex of the left ventricle, thus left ventricular activation initiates and propagates to the right ventricle. This process occurs in the direction of Lead V1, thus the positive QRS polarity and occurs in a direction away from Lead V6, thus the negative QRS polarity.

Fig. 3 is the 12-lead electrocardiogram demonstrating the appearance of PVCs in Leads I, II, III, aVR, aVL and aVF. PVCs are now induced at a higher frequency.

Fig. 4 records the 12-lead electrocardiogram after exactly 28 min of PVCs. Note the striking changes in QRS amplitude in Leads V1 and V6. In Lead V1 the QRS amplitude is still negative, but at a much greater amplitude at $1.9 \mathrm{mV}$. In Lead V6 the QRS amplitude is positive with an amplitude of $0.8 \mathrm{mV}$.

\section{DISCUSSION}

It was possible to demonstrate the Brody effect in the ovine heart, by inducing PVCs in order to reduce the intraventricular volumes. The left ventricle responded as described in other homeotherms (e.g. cats, dogs and chickens) by a reduction in QRS amplitude. As the QRS amplitude in Lead V6 changed from a negative polarity with an amplitude of $1.0 \mathrm{mV}$ to a positive amplitude of $0.8 \mathrm{mV}$, this can be regarded as a reduction of $1.8 \mathrm{mV}$ [0.8 $\mathrm{mV}-(-1.0$ $\mathrm{mV})=1.8 \mathrm{mV}]$.

The right ventricle responded by an increase in QRS amplitude as described by Angelakos \& Gokhan (1963); in this case from $-0.4 \mathrm{mV}$ to $-1.9 \mathrm{mV}$, thus an increase of $1.5 \mathrm{mV}$.

It is concluded that the ovine heart is a valid model for the study of the Brody effect, and, in addition this is also the first report of the Brody effect in the ovine heart. There is a paucity of data on the Brody effect in recent literature and it is still unsatisfactorily explained why there is a different response to a reduction in ventricular volume between the poikilothermic and homeothermic heart. 
The uniqueness of this study is the fact that it is the first study in the literature which documents that PVCs are a valid method of inducing the Brody effect.

\section{REFERENCES}

ANGELAKOS, E.T. \& GOKHAN, N. 1963. Influence of venous inflow volume on the magnitude of the QRS potentials in vivo. Cardiologia, 42:337.

BRODY, D.A. 1956. A theoretical analysis of intracavitary blood mass influence on the heart lead relationship. Circulation Research, 4:731-738.

DANIELS, S., ISKANDRIAN, A,S., HAKKI, A.H., KANE, S.A., BEMIS, C., HOROWITZ, L.N., GREENSPAN, A.M. \& SEGAL, B.L. 1984. Correlation between changes in $\mathrm{R}$ wave amplitude and left ventricular volume induced by rapid atrial pacing. American Heart Journal, 107:711-717.

HORAN, L.G., ANDREAE, R.L. \& YOFFEE, H.F. 1961. The effect of intracavitary carbon dioxide on surface potentials in the intact canine chest. American Heart Journal, 61:504.

ISHIKAWA, K., BERSON, A.S. \& PIPBERGER, H.V. 1971. Electrocardiographic changes due to cardiac enlargement. American Heart Journal, 81:635.

ISHIKAWA, K. 1976. Influence of alterations in the hematocrit upon the P wave and the QRS complex. Chest, 69:762-768.

KER, J \& WEBB, E.C. 2003. Ventriculo-atrial conduction in the ovine heart, caused by premature ventricular complexes. Onderstepoort Journal of Veterinary Research, 70:107-111.

MANOACH, M. 2000. On Brody effect and increase in QRS amplitude. Journal of Cardiovascular Electrophysiology, 11: 833.
MANOACH, M., GITTER, S., GROSSMAN, E. \& VARON, D. 1971. Some considerations regarding the importance of blood, heart and tissue conductivity with regards to QRS amplitude changes after hemorrhage. American Heart Journal, 31:726-728.

MANOACH, M., VARON, D., GROSSMAN, E., GITTER, S. \& SROKA, M. 1971. Influence of bleeding on the QRS amplitude in adult chickens and chick embryo. Israel Journal of Medical Science, 7:708.

MANOACH, M., GITTER, S., GROSSMAN, E., VARON, D. \& GASSNE, S. 1971. Influence of hemorrhage on the QRS complex of the electrocardiogram. American Heart Journal, 82:55-61.

MANOACH, M., GITTER, S., GROSSMAN, E. \& VARON, D. 1972. The relation between the conductivity of the blood and the body tissue and the amplitude of the QRS during heart filling and pericardial compression in the cat. American Heart Journal, 84:72-75.

MYERBURG, R.J. \& KESSLER, K.M. 1998. Recognition, clinical assessment and management of arrhythmias and conduction disturbances, in Hurst's the heart. New York: McGrawHill.

NELSON, C.V., LANGE, R.L., HECHT, H.H., CARLISLE, R.P. \& RUBY, A.S. 1956. Effect of intracardiac blood and of fluids of different conductivities on the magnitude of surface vectors. Circulation, 14:977.

PIPBERGER, H.V., ISHIKAWA, K. \& BERSON. 1972. Reply: Letter to the editor. American Heart Journal, 83:295-297.

SCHAMROTH, L. 1980. Ventricular extrasystoles, ventricular tachycardia and ventricular fibrillation: Clinical electrocardiographic considerations. Progress in Cardiovascular Diseases, 23:13-32.

SCHULTZ, R.A. \& PRETORIUS, P.J. 1972. An electrocardiographic study of normal sheep using a modified technique. Onderstepoort Journal of Veterinary Research, 39:97-106. 